\title{
COMPARACIÓN DE LA RESISTENCIA A LA FRACTURA DE DOS MATERIALES DE REFORZAMIENTO RADICULAR EN DIENTES CON TRATAMIENTO ENDODÓNTICO
}

\author{
COMPARISON OF THE RESISTANCE TO FRACTURE OF TWO ROOT REINFORCEMENT \\ MATERIALS IN TEETH WITH ENDODONTIC TREATMENT
}

Manuel Andrés Palomino-Delgado ${ }^{1}$, Jeanette Allison Gonzales-Mattos ${ }^{2}$, Jhon Torres-Torres ${ }^{3}$, Carmen Rosa García-Rupaya ${ }^{4}$

\section{RESUMEN}

Objetivo: El propósito del estudio fue comparar la resistencia a la fractura de dos materiales de reforzamiento radicular en dientes monorradiculares con tratamiento endodóntico. Metodología: Se incluyeron cuarenta y cinco premolares inferiores humanos recientemente extraídos, los cuales fueron divididos en tres grupos $(n=15)$. El grupo 1 estuvo conformado por dientes con tratamiento de conducto sin ningún tipo de reforzamiento radicular, el grupo 2 estuvo formado por dientes con tratamiento de conducto y reforzados con un cemento a base de silicato de calcio (Biodenti$\left.n e^{T M}\right)$, y el grupo 3 estuvo formado por dientes con tratamiento de conducto que fueron reforzados con un poste de fibra de vidrio. Se evaluó la resistencia a la fractura $(\mathrm{kN})$ utilizando la máquina de ensayo universal, a una velocidad de 0,5 $\mathrm{mm} / \mathrm{min}$ hasta que se produjo la fractura. Se utilizó la prueba de Anova de una vía para comparar la resistencia a la fractura entre los 3 grupos y la prueba de comparaciones múltiples de Tukey. Resultados: Los valores de la media y desviación estándar de cada grupo fueron: grupo 1, gutapercha (GP) $1,01 \pm 0,19 \mathrm{KN}$; grupo 2 , Biodentine (BD) $1,41 \pm 0,25 \mathrm{KN}$, y grupo 3 , postes de fibra de vidrio (PFV) $1,27 \pm 0,16$ KN. Conclusión: El Biodentine ${ }^{T M}$ mostró mejores valores de resistencia a la fractura radicular en comparación con los postes de fibra de vidrio y la gutapercha.

Palabras clave: cemento de silicato de calcio, poste de fibra de vidrio, resistencia a la fractura, fractura radicular

\section{ABSTRACT}

Objective: The aim of this in vitro study was to evaluate the fracture resistance of two root reinforcement materials in monoradicular teeth with endodontic treatment. Methods: Thirty freshly extracted human

Citar como: Palomino-Delgado MA, Gonzales-Mattos JA, Torres-Torres J, Garcia-Rupaya CR. Comparación de la resistencia a la fractura de dos materiales de reforzamiento radicular en dientes con tratamiento endodóntico. Rev Cient Odontol (Lima). 2018; 6 (1): pp. 19-28

\footnotetext{
Especialista en Endodoncia, Magíster en Cariología y Endodoncia.

2 Especialista en Rehabilitación Oral, Docente de posgrado Universidad Norbert Wiener, Lima, Perú.

3 Especialista en Endodoncia, Magíster en Estomatología, docente de posgrado, UNMSM, Lima, Perú.

${ }^{4}$ Especialista en Carielogía y Endodoncia, Magíster en Estomatología, docente de posgrado, Universidad Científica del Sur. Lima, PERÚ.
} 
premolars were divided into three groups $(n=10)$. The teeth in group 1 were endodontically treated without any root reinforcement and a resin restoration was placed in the coronal portion. In group 2 only the apical 5-mm portion of the root canal was filled with guttapercha, and the remaining portion was filled with calcium silicate-based cement (Biodentine $^{\mathrm{TM}}$ ), which was used as root reinforcement material. In group 3 a fiber post was cemented as a root reinforcement material. Fracture resistance $(\mathrm{kN})$ was evaluated using a universal testing machine exerting a compression load at a velocity of $0.5 \mathrm{~mm} / \mathrm{min}$ until fracture occurred. A one-way ANOVA test and Tukey's post-hoc test were used to compare the mean root fracture resistance (kN). Results: The mean values and standard deviation of each group were as follows: Group 1 gutta-percha (GP) $1.01 \pm 0.19 \mathrm{KN}$; Group 2 Biodentine (BD) $1.41 \pm 0.25 \mathrm{KN}$, and; Group 3 fiber post (PFV) $1.27 \pm 0.16 \mathrm{KN}$. Statistically significant differences were not confirmed between the Biodentine (BD) group and fiber post (PFV) group ( $p$ 0.179). Conclusion: Biodentine ${ }^{\mathrm{TM}}$ exhibited better root fracture resistance values compared to fiber post and gutta-percha.

Keywords: Calcium silicate cement, fiber post, fracture resistance, root fracture.

\section{INTRODUCCIÓN}

En la práctica clínica, es común encontrar que piezas dentales que han recibido tratamiento de conducto se fracturen. Esto, muchas veces, se atribuye a que estos dientes, después de una terapia endodóntica, sufren un proceso de deshidratación que los vuelve quebradizos.

Existen diversos factores que afectan la resistencia a la fractura de los dientes tratados endodónticamente, entre los cuales podemos mencionar el tipo de diente, la función, la posición en el maxilar y el espesor de la estructura dental residual, además de la longitud y diámetro del poste (1). Pero la pérdida de estructura dental causada por procedimientos de endodoncia y restauración ha sido principalmente la razón de la fractura de estos dientes tratados (2).

La remoción excesiva de la dentina durante la instrumentación del conducto radicular, la preparación del muñón y los postes pueden causar la pérdida de la resistencia de las raíces y una mayor susceptibilidad a la fractura de la raíz (3-5). Se dice también que los canales radiculares vacíos son más susceptibles a fracturarse que las raíces obturadas, mientras que las raíces reforzadas con materiales intracanales adecuados mantienen resistencia a la fractura (6).

Reeh et al. compararon el efecto de los procedimientos endodónticos y restauradores sobre la rigidez de segundos premolares extraídos y demostraron que los procedimientos endodónticos reducían la rigidez de los dientes un $5 \%$, mientras que los procedimientos restaurativos, como las preparaciones coronales mesio-disto-oclusales, reducen un $60 \%$ la rigidez (7).

Reforzar internamente estos dientes ha sido y es una práctica muy frecuente en odontología. El material de reforzamiento radicular ideal debe tener propiedades físicas similares a las de la dentina (8). La utilización de postes de diversos materiales, como colados, fibra de vidrio y fibra de carbono, han sido utilizados para este fin (9), pero algunos presentan mayor rigidez y dureza que la pieza dentaria, lo que contribuye a aumentar el riesgo de fractura de los dientes tratados endodónticamente.

Algunas resinas compuestas han sido utilizadas para sellar los canales radiculares, con la finalidad de evitar el 
ensanchamiento de las paredes del conducto radicular durante la preparación del poste y aumentar así la resistencia a la fractura $(10,11)$; pero existe una contracción de polimerización que puede comprometer a largo plazo el éxito de la rehabilitación, además de que estos materiales no comparten propiedades similares a la dentina.

Actualmente, existen algunos materiales con base en el silicato tricálcico que han sido propuestos como sustitutos de dentina. Uno de estos materiales es el Biodentine $^{\mathrm{TM}}$, el cual se ha venido utilizando como material para el sellado de perforaciones, en los casos de retrobturaciones en cirugías apicales, recubrimientos pulpares directos e indirectos, y como base de restauraciones (12). Este material presenta un módulo de elasticidad y fuerza compresiva similar a la dentina, por lo que podría ser utilizado como material para el reforzamiento radicular en dientes que han recibido un tratamiento endodóntico y presentan paredes dentinarias remanentes.

Di Fiore y col. realizaron un estudio in vitro con el propósito de evaluar la resistencia a la fractura de las raíces obturadas con conos de gutapercha, resina compuesta (LuxaCore Dual) o cemento con base en silicato de calcio (Biodentine). Se evaluó la resistencia a la fractura en la porción media de cada raíz, utilizando un ensayo de flexión de 3 puntos con una máquina de ensayo universal. Se observó que las raíces de los incisivos anterosuperiores permanentes con tratamiento de conductos obturados con gutapercha, Biodentine o LuxaCore dual tenían resistencia a la fractura similar a la de los dientes que no fueron tratados con endodoncia (13).

Ambica y col. compararon la resistencia a la fractura de dientes tratados endodónticamente con reforzamiento de postes de fibra de vidrio, fibra de carbón y postes experimentales de dentina radicular humana. Los dientes restaurados con postes de dentina exhibieron una mayor resistencia a la fractura y patrones de fractura más favorables que los restaurados con postes de fibra de vidrio y de carbón (9).

Nikhil y col. compararon la resistencia a la fractura de los dientes inmaduros simulados, mediante la colocación de $5 \mathrm{~mm}$ de MTA como barrera apical, y añadieron posteriormente materiales de reforzamiento radicular como postes de fibra de vidrio, postes experimentales de dentina, resina de curado dual Paracore y gutapercha. Concluyeron que los postes de fibra de vidrio, los postes experimentales de dentina y resina de curado dual Paracore fueron capaces de ejercer un efecto de reforzamiento en dientes inmaduros (14).

Dado el contexto señalado, el propósito del estudio fue comparar la resistencia a la fractura de dos materiales de reforzamiento radicular en dientes monorradiculares con tratamiento endodóntico.

\section{MATERIALES Y MÉTODOS}

Se utilizó 45 premolares inferiores donados y recién extraídos por motivos ortodónticos 0 periodontales. Estos fueron desinfectados en una solución de hipoclorito de sodio al $0,5 \%$ y se almacenaron en solución salina fisiológica.

Los dientes fueron inspeccionados con un microscopio quirúrgico a una magnificación de $5 x$, con la finalidad de detectar abrasiones, grietas o fracturas. Posteriormente, se realizó una radiografía periapical de cada diente para visualizar su anatomía interna. Los dientes que presentaron alguna variación anatómica fueron excluidos del estudio. 
Para calcular el tamaño muestral se utilizó una fórmula de comparación de medias con un nivel de confianza del $95 \%$ y poder estadístico del $80 \%$, y se obtuvo un tamaño muestral de quince por cada grupo con una muestra total de 45 premolares.

Los dientes fueron decoronados y las raíces estandarizadas a $14,5 \mathrm{~mm}$, medidas con un calibrador digital. Seguidamente, se introdujo una lima de acero inoxidable Dentsply Maillefer $n .^{\circ} 10$ hasta observarla por el foramen apical; a esta medida se le restó $1 \mathrm{~mm}$ y se obtuvo así la longitud de trabajo. Para la preparación se utilizó una técnica Crown Down con instrumentos rotatorios de níquel titanio Pro Taper Universal (Dentsply Maillefer) hasta el instrumento F3 a la longitud de trabajo. Entre cada instrumento se procedió a realizar una irrigación con hipoclorito de sodio al 5,25\%, con una jeringa de $10 \mathrm{ml}$ y una aguja de irrigación Navitip calibre 30 de $25 \mathrm{~mm}$ Ultradent (EE. UU.). Finalmente, se irrigó con EDTA $17 \%$ de Biodinámica (Brasil) por un minuto, y luego con hipoclorito de sodio al $5,25 \%$.

Los dientes fueron obturados con la técnica de onda continua y cemento de obturación resinoso AH 26 (Dentsply). Posteriormente, se los almacenó en solución salina fisiológica inmediatamente después de la obturación del conducto radicular.

Se realizó un muestreo aleatorio simple y se dividieron las muestras en tres grupos. En el grupo 1 estuvieron los dientes con tratamiento de conducto obturados, pero sin ningún tipo de reforzamiento, solo con la gutapercha hasta la unión cementoesmalte (figura 1). El grupo 2 se desobturó hasta dejar $5 \mathrm{~mm}$ apicales, se lavó el canal radicular con suero fisiológico, se secó con puntas de papel absorbente y se colocó cemento con base en silicato tricálcico Biodentine ${ }^{\mathrm{TM}}$ (Septodont), el

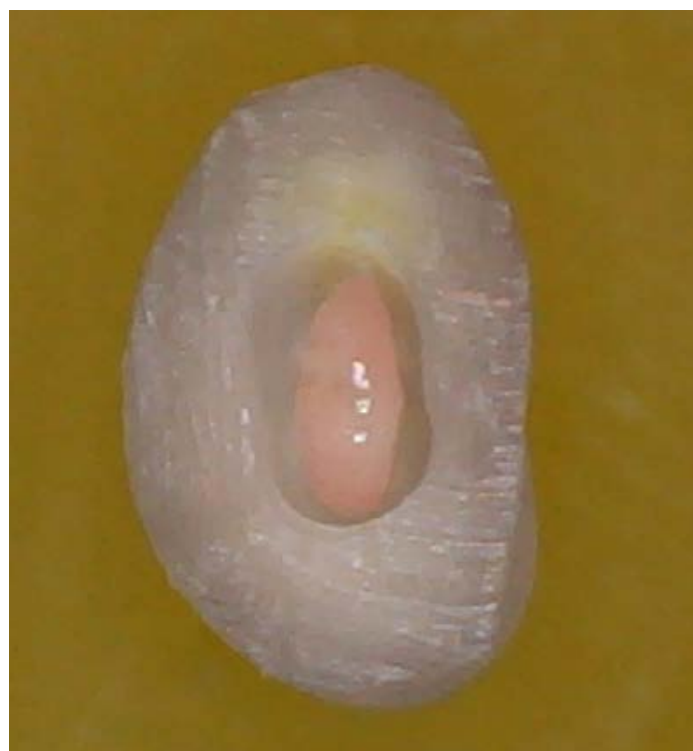

FIGURA 1. GRUPO 1: GUTAPERCHA.

cual fue preparado de acuerdo con las instrucciones del fabricante y colocado en pequeños incrementos, seguido por la compactación vertical de cada incremento hasta que el cemento obturó la totalidad del conducto y la cavidad de acceso (figura 2). En el caso del grupo 3 , se utilizó un poste de fibra de vidrio de diámetro 1 (Angelus), realizándose la preparación para la colocación de poste de fibra de vidrio utilizando fresas Peeso \#3, y se dejaron $5 \mathrm{~mm}$ apicales del mate-

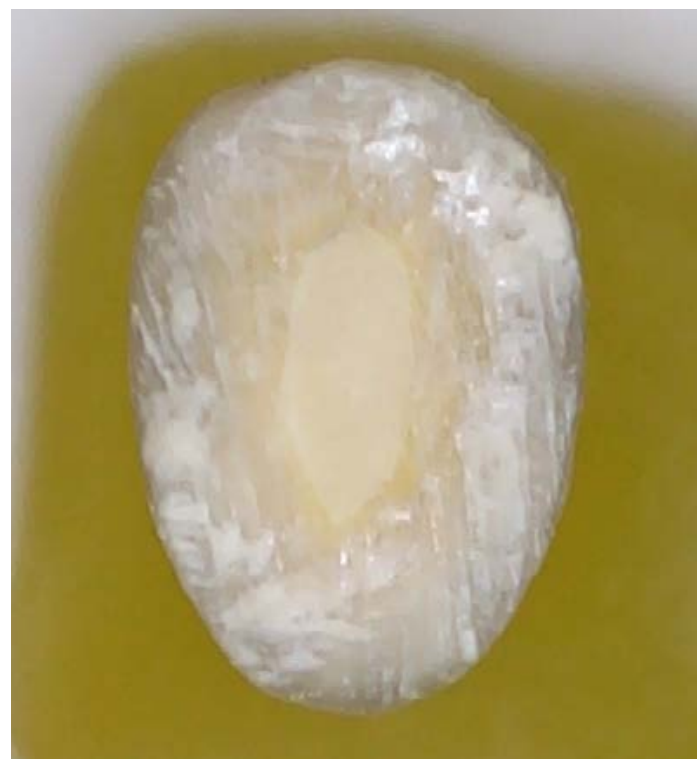

Figura 2. GRUPo 2: Biodentine. 


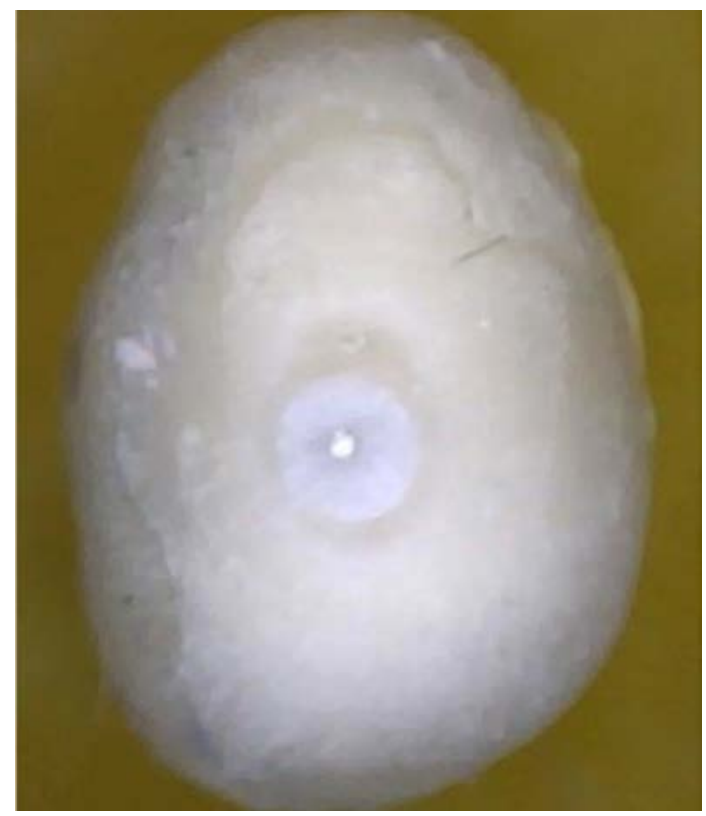

FIGURA 3. GRUPO 3: POSTES DE FIBRA DE VIDRIO.

rial de obturación. Se lavó el espacio del conducto radicular con alcohol de $70^{\circ}$, se secó con puntas de papel absorbente y, posteriormente, se colocó un cemento autograbante U200 (3M ESPE) con puntas Smart tips en el espacio del canal radicular. A continuación, se insertó el poste de fibra de vidrio y se fotopolimerizó por 40 segundos (figura 3).

Luego de los procedimientos de refuerzo radicular, se procedió a grabar la cavidad de acceso con ácido ortofosfórico al $37 \%$, durante 15 segundos; se lavó la cavidad con agua y se secó con papel absorbente. A continuación, se colocó el adhesivo Single Bond (3M ESPE) con una microbrocha y se activó por 20 segundos con una lámpara de fotocurado Elipar (3M), para después colocar una resina compuesta Z350 (3M ESPE) en la cavidad de acceso. Este procedimiento fue repetido en los tres grupos. Posteriormente, se tomó una radiografía periapical para verificar el relleno del canal radicular.

Se fabricó un bloque de acrílico para proporcionar una adecuada estabilidad durante la prueba y, a su vez, para usarlo como soporte de la simulación del ligamento periodontal. Las superficies de las raíces se sumergieron en cera roja derretida hasta 2,0 mm por debajo de la unión cemento-esmalte, lo que resultó en una capa delgada de cera en un cilindro de plástico con $25 \mathrm{~mm}$ de diámetro. El diente se ubicó en el centro y, luego, se vertió acrílico autopolimerizable. Después de la polimerización del acrílico, los dientes fueron retirados del cilindro. Se eliminó la cera de la superficie de la raíz y del cilindro de acrílico, el cual simuló el alveolo del diente. Seguidamente, se llenó el alveolo con silicona por adición polivinilsiloxano Silagum DMG y se insertó el diente en el alveolo de acrílico, los excesos se eliminaron con una hoja de bisturí; posteriormente, los dientes se almacenaron a un $100 \%$ de humedad.

Para la prueba de resistencia a la fractura, se utilizó la máquina de ensayo universal a una velocidad de $0,5 \mathrm{~mm} / \mathrm{min}$ y la carga fue aplicada de forma vertical con respecto al eje longitudinal del diente hasta fracturarse (figura 4). Las medidas fueron registradas en kilonewtons. Para el análisis de los resultados se utilizó la prueba Anova de una vía y la prueba de comparaciones múltiples de Tukey.

TABLA 1. DESCRIPCIÓN DE LOS VALORES DE LA RESISTENCIA A LA FRACTURA DE DOS MATERIALES DE REFORZAMIENTO RADICULAR EN DIENTES MONORADICULARES CON TRATAMIENTO ENDODÓNTICO

\begin{tabular}{|l|c|c|c|c|c|c|c|}
\hline Grupos de estudio & $n$ & Media & D.E. & Mediana & min & máx & Varianza \\
\hline Gutapercha & 15 & 1,007 & 0,197 & 0,988 & 0,77 & 1,48 & 0,039 \\
\hline Biodentine & 15 & 1,407 & 0,249 & 1,337 & 0,88 & 1,75 & 0,062 \\
\hline Postes de fibra de vidrio & 15 & 1,270 & 0,164 & 1,310 & 0,87 & 1,60 & 0,027 \\
\hline
\end{tabular}

Valores expresados en kilonewton 


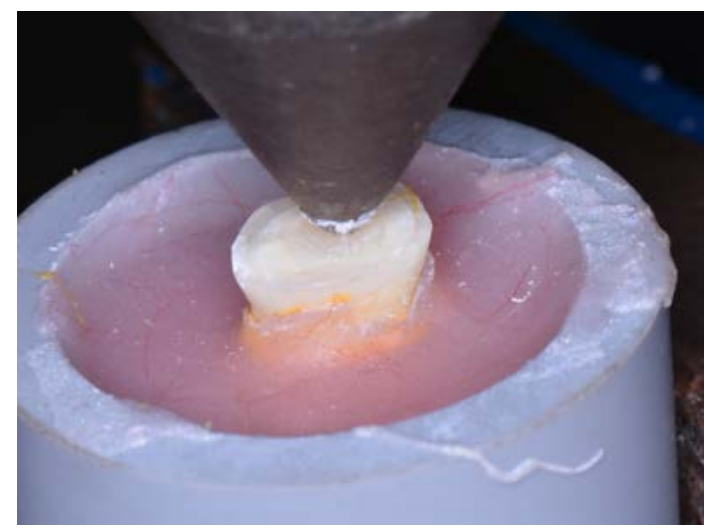

Figura 4. Prueba de Resistencia a la fractura DE MÁQUINA DE ENSAYO UNIVERSAL.

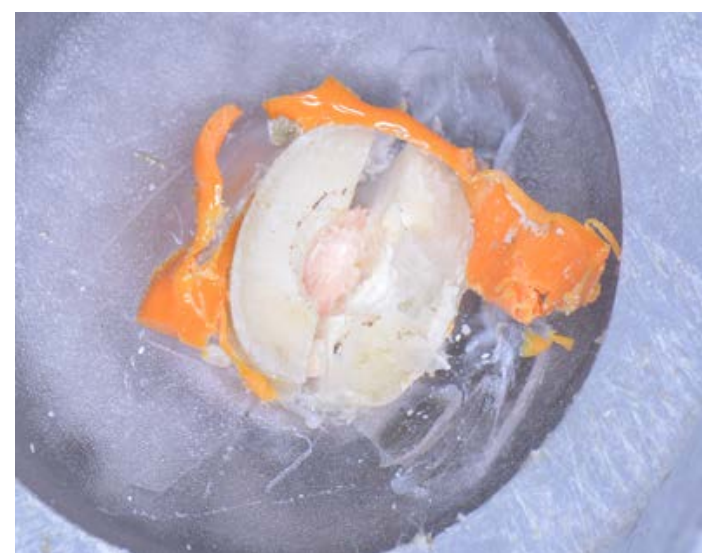

FIGURA 5. Fractura dEL GRUPO1: GUTAPERCHA.

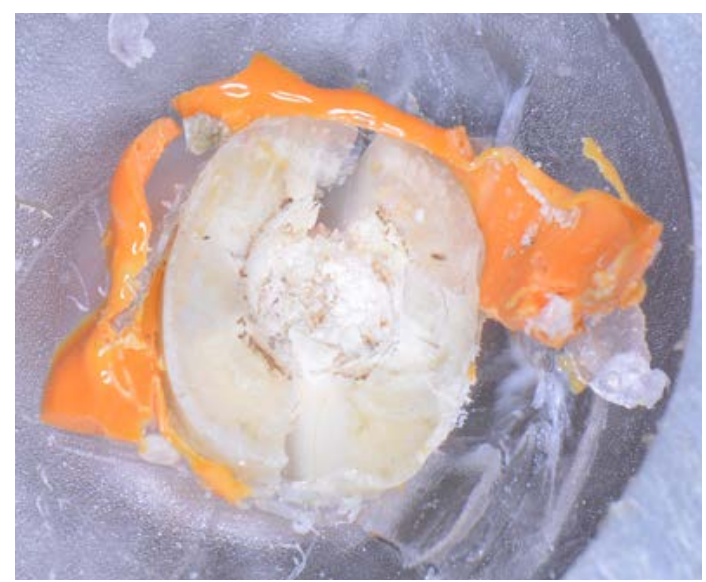

Figura 6. Fractura dEL GRUPO 2: Biodentine.

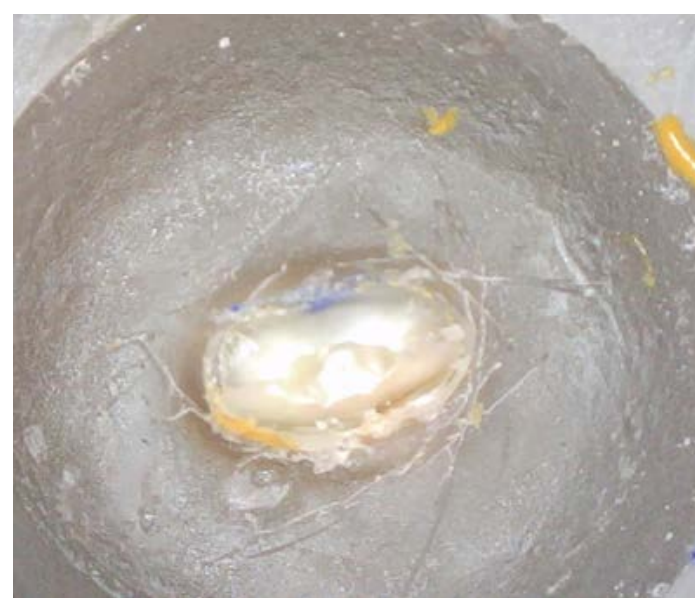

Figura 7. Fractura dEL GRUPO 3: POSTE DE FIBRA DE VIDRIO.

\section{RESULTADOS}

El valor promedio de la resistencia a la fractura de cada grupo se observa en la tabla 1. El Biodentine ${ }^{\mathrm{TM}}$ presentó mayor resistencia a la fractura que los postes de fibra de vidrio; sin embargo, no se observó diferencias estadísticamente significativas $(p=0,179)$ (tabla 2$)$.

\section{DISCUSIÓN}

Este estudio in vitro comparó la resistencia a la fractura de dos materiales de reforzamiento radicular en dientes monoradiculares con tratamiento endodóntico. Los materiales probados fueron el poste de fibra de vidrio y un cemento hecho de silicato de calcio (figura 5, 6 y 7). Existen pocos estudios que comparen estos materiales como reforzamiento radicular y ninguno con la fuerza aplicada de forma perpendicular, siguiendo el eje longitudinal de la raíz. El presente estudio trató de reproducir las condiciones en las que se encuentra la raíz, simulando el alveolo con acrílico de autopolimerizado y el ligamento periodontal con silicona por adición.

Murtuza y col. (15) midieron la resistencia a la fractura inmediatamente y después 
TABLA 2. COMPARACIÓN DE LA RESISTENCIA A LA FRACTURA DE DOS MATERIALES DE REFORZAMIENTO RADICULAR EN DIENTES MONORADICULARES CON TRATAMIENTO ENDODÓNTICO

\begin{tabular}{|c|c|c|c|c|c|c|}
\hline & \multicolumn{6}{|c|}{ IC $95 \%$} \\
\hline Grupos de estudio & $\mathrm{n}$ & Media \pm D.E. & Inferior & Superior & Valor $\mathrm{p} 1$ & Valor p2 \\
\hline G1 Gutapercha & 15 & $1,007 \pm 0,197$ & 0,77 & 1,48 & & G1 vs G2 $=<0,001^{\star *}$ \\
\hline G2 Biodentine & 15 & $1,407 \pm 0,249$ & 0,88 & 1,75 & $<0,001^{*}$ & G2 vs G3 $=0,179 * *$ \\
\hline G3 Postes de fibra de vidrio & 15 & $1,270 \pm 0,164$ & 0,87 & 1,60 & & G1 vs G3 $=0,003^{* *}$ \\
\hline
\end{tabular}

de tres meses de aplicado el material de reforzamiento radicular, y obtuvieron resultados que están muy por debajo de los obtenidos en este estudio. Esto probablemente se deba a que en esa investigación utilizaron dientes inmaduros simulados, los cuales sufren un desgaste de la pared interna del conducto radicular. No encontraron diferencias estadísticamente significativas entre la resina dual Paracore y el Biodentine, lo que coincide con el presente estudio.

Di Fiore y col. (13) compararon gutapercha, Biodentine y Luxacore, aplicando la fuerza en la raíz del diente, pero esta estuvo posicionada de forma horizontal. Encontraron que no había diferencia significativa en cuanto a la resistencia a la fractura entre estos materiales (13). En el presente estudio, la fuerza fue aplicada perpendicular al eje longitudinal del diente, lo que simularía de una forma más realista las fuerzas producidas en boca; sin embargo, los resultados coinciden con Di Fiore puesto que no hubo diferencias entre el entre Biodentine y los postes de fibra de vidrio.

Aunque la comparación directa no es aplicable por no usar los mismos materiales, se analizaron algunos trabajos que emplean la dentina humana como material de reforzamiento radicular, debido a no encontrar antecedentes que comparen estos materiales. El Biodentine se presenta como un sustituto de la dentina por las propiedades similares que presenta, por lo cual se podría sugerir su utilización como material para el reforzamiento radicular.

Ambica y col. compararon la resistencia a la fractura de dientes tratados endodónticamente con reforzamiento de postes de fibra de vidrio, fibra de carbón y postes experimentales de dentina radicular humana, y encontraron que los dientes restaurados con postes de dentina exhibieron una mayor resistencia y los patrones de fractura fueron más favorables que con los postes de fibra de vidrio y de carbón (9). Nikhil y col. compararon la resistencia a la fractura de los dientes inmaduros simulados. Para ello, colocaron $5 \mathrm{~mm}$ de MTA como barrera apical y utilizaron como materiales de reforzamiento radicular postes de fibra de vidrio $1164.6 \mathrm{~N}$, postes experimentales de dentina $994.4 \mathrm{~N}$, resina curado dual Paracore $873.8 \mathrm{~N}$. El resultado mostró una diferencia significativa entre estos materiales, concluyendo que los postes de fibra de vidrio y los postes experimentales de dentina son capaces de ejercer un efecto de refuerzo en los dientes inmaduros simulados (14). En el presente estudio, el Biodentine mostró una mayor resistencia a la fractura $1407 \mathrm{~N}$ que los postes de fibra de vidrio, aunque no hubo una diferencia significativa. 


\section{CONCLUSIONES}

El Biodentine ${ }^{\mathrm{TM}}$ mostró mejores valores de resistencia a la fractura radicular en comparación con los postes de fibra de vidrio y la gutapercha, pero no hubo una diferencia estadísticamente significativa, lo cual demuestra que el Biodentine ${ }^{\mathrm{TM}}$ presentó un comportamiento similar a los postes de fibra de vidrio en cuanto a su resistencia.

\section{REFERENCIAS BIBLIOGRÁFICAS}

1. Vallejo M, Maya C, Erazo N. Resistencia a la fractura de dientes con debilitamiento radicular. Rev CES Odont 2011; 24 (1): 59-69.

2. Kishen A. Mechanisms and risk factors for fracture predilection in endodontically treated teeth. Endodontic Topics 2006; 13: 57-83.

3. Guzy GE, Nicholls JI. In vitro comparison of intact endodontically treated teeth with and without endo-post reinforcement. J Prosthet Dent 1979; 42: 39-44.

4. Trope M, Maltz DO, Tronstad L. Resistance to fracture of restored endodontically treated teeth. Endod Dent Traumatol 1985; 1: 108-11.

5. Trabert KC, Caput AA, Abou-Rass M. Tooth fractured a comparison of endodontic and restorative treatments. J Endod 1978; 4: 341-5.

6. Sornkul E, Stannard JG. Strength of roots before and after endodontic treatment and restoration. J Endod 1992; 18: 440-3.

7. Reeh ES, Messer HH, Douglas WH. Reduction in tooth stiffness as a result of endodontic and restorative procedures. J Endod 1989; 15: 512-6.

8. Cheung W. A review of the management of endodontically treated teeth. Post, core and the final restoration. J Am Dent Assoc 2005; 136: 611-9.

9. Ambica K, Mahendran K, Talwar S, Verma M, Padmini G, Periasamy R. Comparative evaluation of fracture resistance under static and fatigue loading of endodontically treated teeth restored with carbon fiber posts, glass fiber posts, and an experimental dentin post system: an in vitro study. J Endod 2013; 39: 96-100.

10. Balkaya MC, Birdal IS. Effect of resin-based materials on fracture resistance of endodontically treated thin-walled teeth. J Prosthet Dent 2013; 109: 296-303.

11. Scotti N, Rota R, Scansetti M, Paolino DS, Chiandussi G, Pasqualini D et al. Influence of adhesive techniques on fracture resistance of endodontically treated premolars with various residual wall thicknesses. J Prosthet Dent 2013; 110: 376-82.

12. Rajasekharan S, Martens LC, Cauwels RG, Verbeeck RM. Biodentine material characteristics and clinical applications: a review of the literature. Eur Arch Paediatr Dent 2014; 15 (3): 147-58. 
13. Di Fiore P, Reyes A. Evaluation of a calcium silicate-based cement as a root reinforcement material for endodontically treated maxillary anterior teeth. J Prosthet Dent 2016; 115: 35-41.

14. Nikhil V, Jha P, Aggarwal A. Comparative evaluation of fracture resistance of simulated immature teeth restored with glass fiber posts, intracanal composite resin, and experimental dentine posts. Scientific World Journal 2015: 1-5.

15. Zhabuawala MS, Nadig RR, Pai VS, Gowda Y. Comparison of fracture resistance of simulated immature teeth with an open apex using Biodentine and composite resin: An in vitro study. J Indian Soc Pedod Prev Dent 2016; 34: 377-82.

16. Zamin C, Silva-Sousa YT, Souza-Gabriel AE, Messias DF, Sousa-Neto MD. Fracture susceptibility of endodontically treated teeth. Dental Traumatology 2012; 28: 282-6.

17. Torres LM, Torres C. Characterization of endodontically treated dentin: a review. Rev Fac Odontol Univ Antioq 2014; 25 (2): 372-88.

18. Fuentes M. Propiedades mecánicas de la dentina humana. Av Odontoestomatol 2004; 20 (2): 79-83.

19. Singh $\mathrm{H}$, Kaur M. Biodentine: a promising dentin substitute. J Interdiscipl Med Dent Sci 2014; 2: 1-5.

20. Priyalakshmi S, Ranjan M. Review on biodentine-a bioactive dentin substitute. IOSR Journal of Dental and Medical Sciences 2014; 13 (1): 13-7.

21. Malkondu O, Karapinar M. A Review on biodentine, a contemporary dentine replacement and repair material. BioMed Research International 2014; 1-10.

22. Grech L, Mallia B, Camilleri J. Investigation of the physical properties of tricalcium silicate cement-based root-end filling materials. Dental Materials 2013; 29 (2): 20-8.

23. Elnaghy AM. Influence of acidic environment on properties of biodentine and white mineral trioxide aggregate: a comparative study. J Endod 2014; 40: 953-7.

24. Baybora M, Hossein M, McCann A. Effect of acid etching procedures on the compressive strength of 4 calcium silicate-based endodontic cements. J Endod 2013; 39: 1646-8.

25. Camilleri J. Investigation of Biodentine as dentine replacement material. Journal of Dentistry 2013; 41 (7): 600-10.

26. Laurent $\mathrm{P}$, Camps J, About I. Biodentine induces TGF-beta1 release from human pulp cells and early dental pulp mineralization. Int Endod J 2012; 45: 439-48.

27. Zhou H, Shen $Y$, Wang Z. In vitro cytotoxicity evaluation of a novel root repair material. J Endod 2013; 39: 478-83.

28. Luo Z, Li D, Kohli M. Effect of Biodentine on the proliferation, migration and adhesion of human dental pulp stem cells. Journal of Dentistry 2014; 42 (4): 490-7. 
Manuel Andrés Palomino-Delgado, Jeanette Allison Gonzales-Mattos,

Jhon Torres-Torres, Carmen Rosa García-Rupaya

29. Soares C, Gava Pizi E. Influence of root embedment material and periodontal ligament simulation on fracture resistance tests. Braz Oral Res 2005; 19 (1): 11-6.

30. Sagsen B, Zortuk M. In vitro fracture resistance of endodontically treated roots filled with a bonded filling material or different types of posts. J Endod 2013; 39: 1435-7.

31. Sawyer A, Nikonov S, Pancio A. Effects of calcium silicate-based materials on the flexural properties of dentin. J Endod 2012; 38: 680-3.

CorRESPONDENCIA: mjyl42@ hotmail.com

RECIBIDO: 23/04/2018

ACEPTADO: 23/05/2018 